\title{
INVESTIGACIONES
}

\section{Geografías de la investigación académica sobre migración y escuela: voces, silencios y prospectivas de nuestra profesión*}

\author{
Geographies of academic research on migration and school: \\ voices, silences and prospects of our profession
Felipe Jiménez Vargas ${ }^{a}$, René Valdés Morales ${ }^{b}$ Magdalena Aguilera Valdiviac \\ a Pontificia Universidad Católica de Valparaíso \\ Correo electrónico: felipe.jimenez@pucv.cl \\ ${ }^{\text {b }}$ Pontificia Universidad Católica de Valparaíso \\ Correo electrónico: revalmorales@gmail.com \\ ${ }^{\mathrm{c}}$ Universidad de Santiago de Chile \\ Correo electrónico: maria.aguilera.v@usach.cl
}

\begin{abstract}
RESUMEN
Pese al progresivo interés por desarrollar investigación educativa sobre la escolarización de estudiantes inmigrantes, a la fecha no existe una revisión comprehensiva respecto a qué contribuciones hemos realizado desde el ámbito académico al estudio del fenómeno migratorio en el ámbito escolar. En ese escenario y mediante el análisis de 32 artículos, documentamos la voz académica sobre la escolarización de estudiantes inmigrantes, permitiendo responder cuatro preguntas claves: 1) cuál es la naturaleza de estos artículos, 2) sobre qué tópicos se ha investigado, 3) qué metodologías han predominado en aquellos de naturaleza investigativa y, finalmente, 4) qué enfoques de educación intercultural hay a la base de nuestros trabajos. Las discusiones apuntan a identificar los aportes así como los desafíos que enfrenta el campo con relación a las preguntas antes señaladas. El propósito de nuestro trabajo es por tanto contribuir a una mayor auto-comprensión de la investigación educativa realizada en contextos de migración.
\end{abstract}

Palabras claves: revisión de literatura, inmigración, educación intercultural, investigación educativa

\section{ABSTRACT}

In spite of the progressive interest in developing educational research on the schooling of immigrant students, to date there is no comprehensive review regarding what contributions we have made from the academic field to the study of the migratory phenomenon in the school environment. In this scenario and through the analysis of 32 articles, we document the academic voice on the schooling of immigrant students, which allow us to answer four key questions: 1) what is the nature of these articles? 2) what topics have been investigated? 3) what methodologies have predominated in those of an investigative nature? and finally, 4) what intercultural education approaches are based on them? The discussions aim to identify the contributions as well as the challenges facing the field in relation to the questions mentioned above. The purpose of our work is therefore to contribute to a greater self-understanding of educational research carried out in migration contexts.

Key words: literature review, immigration, intercultural education, educational research 
Estudios Pedagógicos XLIV, N 3: 173-191, 2018

GEOGRAFÍAS DE LA INVESTIGACIÓN ACADÉMICA SOBRE MIGRACIÓN Y ESCUELA: VOCES, SILENCIOS Y

PROSPECTIVAS DE NUESTRA PROFESIÓN

\section{EL ESTADO ACTUAL DEL FENÓMENO MIGRATORIO EN EL ÁMBITO ESCOLAR CHILENO}

Si bien el fenómeno migratorio en las escuelas es relativamente reciente, de acuerdo a las cifras oficiales existen más de 61 mil estudiantes de origen inmigrante escolarizados en Chile ${ }^{1}$. Sin embargo, sabemos que es una cifra aparente ya que la implementación del Programa de Regularización impulsado por el Ministerio del Interior y Ministerio de Educación, ha significado actualizar en más de 20 mil dichas cifras. Considerando que es una aplicación piloto, el número total de estudiantes inmigrantes podrían superar este 2017 los 100 mil inmigrantes.

Ahora bien, convendría hacer dos alcances a estas cifras con tal de hacerlas más transparentes. Primero, estas han ido aumentado progresiva y significativamente en el transcurso de las últimas dos décadas: en los últimos once años se ha sextuplicado el número de estudiantes de origen extranjero (Joiko \& Vásquez, 2016). Si para el año 2011 existía un promedio de siete estudiantes inmigrantes por escuela en Chile, el año 2016 la cifra aumentó a once (Villalobos \& Carrillo, 2014). Y segundo, la distribución de estos estudiantes no se ha producido de manera equilibrada en el sistema educativo, sino que se ha concentrado mayoritariamente (56\%) en escuelas municipales (Joiko y Vásquez, 2016). Esto ha tenido como resultado que en determinadas escuelas públicas la matrícula de inmigrantes supere el cuarenta, cincuenta e incluso el sesenta por ciento de estudiantes.

Estas transformaciones si bien han ido acompañadas de investigaciones educativas que buscan comprender y explicar las diferentes problemáticas y desafíos que suponen, no ha habido a la fecha un ejercicio de sistematización que permita ofrecer una panorámica global y exhaustiva de la contribución realizada desde el ámbito académico. De acuerdo a Joiko y Vásquez (2016), las investigaciones en torno a educación y migración se han concentrado principalmente en el estudio de las microinteracciones escolares, las relaciones familia-escuela y la relación migración-escuela con el enfoque de educación intercultural. Más recientemente, Stefoni \& Stang (2017) identifican cuatro nudos temáticos en torno a estudios sobre migración en Chile, siendo el ámbito educativo el segundo de ellos y aquel que concentra una parte importante de los estudios en torno a discriminación y racismo.

A modo de ejemplo, la revisión hecha por Grant \& Sleeter (1985) en el plano internacional sobre más de 200 artículos sobre educación multicultural permitieron a sus autores no sólo tener una mirada comprehensiva sobre los patrones y tendencias de lo escrito, sino fundamentalmente en detectar las debilidades en las formas de hacer investigación. Algo similar puede concluirse sobre el trabajo de Jiménez en torno a educación intercultural en Chile, que además de identificar las principales fuentes de diversidad estudiadas - pertenencia étnica, nacionalidad y discapacidad-, evidencia el predominio de perspectivas interculturales centradas en el mantenimiento de la identidad cultural y el fortalecimiento de las relaciones humanas. Con estas y otras revisiones como referentes (Cisternas, 2011; Gorski, 2009), el propósito de este trabajo es amplificar las revisiones existentes, lo que permitirá contar con una mayor caracterización y una mayor claridad de los desafíos en materia investigativa. Esperamos a fin de cuentas que nuestro mapeo contribuya a la toma de decisiones del ámbito académico a la hora de definir sobre qué investigar, cómo investigar y desde qué lugar hacerlo.

Datos solicitados al Ministerio de Educación por el portal Transparencia Chile. 


\section{ORGANIZANDO EL MODO DE ANALIZAR LA PRODUCTIVIDAD ACADÉMICA}

Realizamos una búsqueda sistemática de artículos en bases de datos indexadas -WoS, Scopus, Scielo, Redalyc y Latindex- a través del uso de descriptores. Los criterios de selección de los documentos fueron: que remitieran a trabajos teóricos o empíricos sobre la realidad educativa chilena, referidos a niveles de educación obligatoria y que abordaran alguna dimensión y/o temática de la relación migración y escuela. La búsqueda arrojó un total de 32 artículos.

\section{RESULTADOS}

Para responder las preguntas centrales de este trabajo -qué se ha investigado, cómo se ha hecho, desde qué lugar, y qué implicancias tiene este escenario-, presentamos los resultados agrupados en cuatro categorías emergentes del análisis (Glaser \& Strauss, 1967)2: naturaleza de los documentos, abordajes metodológicos, tópicos de estudio y enfoques presentes.

Antes de presentar las categorías, un primer análisis cronológico demuestra con claridad el crecimiento sostenido y exponencial de la productividad en relación al fenómeno migratorio en el ámbito educativo. Si para el período 2005-2008 se publicaron solo 2 artículos, entre los años 2009 y 2012 fueron 12, mientras que para el período 20132016 se publicaron 18 artículos; esto supone un incremento del $800 \%$ entre el primer y tercer período.

\subsection{EL FORMATO DE ESCRITURA: LA NATURALEZA DE LOS ARTÍCULOS}

De los 32 artículos revisados comprendidos entre los años 2006 y 2016, 11 tienen una naturaleza teórico-ensayística mientras que los 19 restantes, corresponden a resultados de investigaciones por lo que son de naturaleza empírico-investigativa. De forma residual, solo 2 artículos reportan experiencias pedagógicas implementadas en escuelas con estudiantes inmigrantes, las que serán comentadas al final del apartado 3.3 en torno a tópicos.

Con relación al corpus teórico-ensayístico no es posible establecer la inclinación hacia algún tema por sobre otros, existiendo un corpus que abordan de manera transversal los principales tópicos descritos más adelante: formación inicial, representaciones sociales así como sistematizaciones de modelos de gestión de la diversidad con relación a la escolarización. Un ejemplo es el trabajo realizado por Bravo (2012) que a través de un análisis de las representaciones sociales en torno a la cualidad migratoria en el espacio escolar, aboga por la necesidad de inculcar una pedagogía del reconocimiento cultural. Muy diferente al anterior, el trabajo de Carillo (2014) aporta una reflexión epistemológica en torno a las construcciones simbólicas hechas por docentes en torno a inclusión educativa.

La situación de los artículos empírico-investigativos es distinta ya que a diferencia del corpus anterior, sí concentra mayoritariamente estudios en torno a las diversas disposiciones

Para el caso de la cuarta categoría enfoques que supuso un protocolo analítico más prestablecido, el procedimiento consistió en: a) definir conceptualmente las siete categorías utilizadas; b) lectura de los artículos y codificación de citas textuales; c) revisión por parte del equipo investigador del listado de citas asociadas a las diferentes categorías; y d) selección final de cuarenta citas textuales distribuidas en las siete categorías predefinidas. 
que actores educativos presentan hacia el fenómeno migratorio y/o estudiantes inmigrantes. El trabajo de Stefoni, Stang y Riedemann (2016) ejemplifica el ámbito al develar las actitudes de invisibilización y naturalización de la discriminación cultural por parte de ciertos profesionales de la escuela, y de las barreras que esto introduce para el desarrollo de una auténtica educación en clave intercultural. De igual forma, el estudio de Hernández (2016) visibiliza por ejemplo las tensiones curriculares que experimentan las escuelas a propósito de la llegada de estudiantes inmigrantes así como las estrategias utilizadas para intentar resolverlas.

\subsection{EL CÓMO DE LA INVESTIGACIÓN: DECISIONES METODOLÓGICAS TOMADAS}

Como una forma de profundizar en los resultados de la categoría anterior, nos propusimos realizar un zoom analítico en aquellos artículos de naturaleza empírico-investigativa con el fin de ofrecer una caracterización aún más fina de las investigaciones sobre migración y escuela, y la unidad de estudio por tanto de este trabajo. Una caracterización más fina del corpus analizado permitirá a fin de cuentas contar con mayores antecedentes a la hora de plantear los principales desafíos de este ámbito de estudio, así como mejores argumentos a la hora de plantear nuestras recomendaciones en la materia.

De los 19 artículos de naturaleza empírico-investigativa, poco más de la cuarta parte (5) corresponden a trabajos con metodologías cuantitativas y los otros dos tercios (13) a metodologías cualitativas. A la fecha solo un estudio publicado articula ambas perspectivas.

Profundizando en el ámbito cuantitativo podemos apreciar que las investigaciones incorporan el uso de instrumentos estandarizados en la producción de datos, y esto lo hacen mayoritariamente para cuantificar las disposiciones educativas hacia la migración y/o inmigrantes en el ámbito escolar. Es así por ejemplo que el estudio de Navas y Sánchez (2010), a través del uso de una escala de actitudes hacia la educación multicultural, concluyen que los estudiantes de pedagogía de las tres universidades chilenas participantes no presentan niveles preocupantes de prejuicio manifiesto. Si bien llaman la atención de los investigadores las diferencias encontradas asociadas al género, la naturaleza de la investigación no permite, no obstante, hacer algún tipo de afirmación concluyente al respecto.

Con relación al ámbito cualitativo el análisis evidencia que hay una significativa predominancia de investigaciones cuyos enfoques son de aproximación etnográfica, en la medida que incorporan dentro de sus técnicas de producción de datos la observación/ participación directa en los contextos de estudio y el empleo de diarios de campo. Es asî como el trabajo de Donoso, Mardones y Contreras (2009) realiza un estudio de caso con una de las primeras escuelas públicas que en Santiago comenzó a escolarizar masivamente estudiantes inmigrantes. Con una caracterización del contexto geográfico y a través de una descripción densa del trabajo de campo realizado mediante entrevistas y observaciones participantes, los autores muestran el recorrido trazado por la propia escuela como forma de dar respuesta a las necesidades de estos estudiantes y familias, así como identificar los logros y desafíos pendientes en este proceso. Sin embargo, y el estudio mencionado da cuenta de ello, lo etnográfico es entendido por la mayoría de estas investigaciones como algo que remite a las técnicas de producción empleadas y no como algo que remite al enfoque teórico-metodológico que debiese guiar una investigación. Esto hace que no sea posible encontrar en las investigaciones revisadas dos de los principales criterios demarcatorios de 
la etnografía, que son la centralidad del concepto de cultura en la investigación educativa (Wolcott, 1993) y la explicación holístico-ecológica de los fenómenos educativos estudiados (Ogbu, 1993; Serra, 2004). Esto último es particularmente relevante ya que muchas de las explicaciones que las investigaciones dan a las problemáticas escolares, no consideran con suficiente fuerza el rol que determinadas condiciones estructurales -de desigualdad- del tejido social, tienen en la génesis o reproducción de problemáticas socioeducativas que afectan a estudiantes pertenecientes a grupos sociales desfavorecidos (Sleeter, 2013).

Desde una perspectiva mixta, el trabajo de Abett de la Torre (2011) en torno percepciones de estudiantes migrantes sobre la multiculturalidad de sus escuelas y de su propio proceso de incorporación a ellas, combina el uso de un cuestionario de asociación libre de términos con la posterior realización de entrevistas en profundidad.

\subsection{EL QUÉ DE LA INVESTIGACIÓN SOBRE MIGRACIÓN Y ESCUELA: LOS TÓPICOS DE ESTUDIO}

A lo largo del período analizado la investigación académica se condensa en tres conglomerados temáticos ${ }^{3}:$ 1) disposiciones docentes, 2) discriminación escolar y 3) gestión de la diversidad. A diferencia del ámbito anterior hemos realizado el análisis con la totalidad de documentos consultados -teóricos (11), empíricos (19) y experiencias (2)-, ya que independiente de la naturaleza de los documentos, todos ellos reflejan de una u otra forma las voces académicas aquí examinadas.

En cuanto al conglomerado de disposiciones docentes, entendidas como tendencias para actuar de una particular manera bajo circunstancias específicas (Villegas, 2007), encontramos investigaciones que abordan representaciones sociales, actitudes, expectativas, prejuicios y estereotipos de actores educativos ante la llegada de los estudiantes inmigrantes a la escuela. Este primer conglomerado apela a que las posturas de los profesionales de la escuela frente a los inmigrantes se relaciona con la valoración que tengan las escuelas hacia una educación intercultural, dando a entender que aspectos como prejuicios o actitudes negativas son barreras importantes para atender a la diversidad (Abett de la Torre, 2011; Aranda, 2011; Aranda \& Castro, 2007; Bravo, 2012; Cárdenas; 2006; Carrillo, 2014; González, Berríos \& Buxarrais, 2013; Moreno \& Oyarzún, 2013; Navas, Holgado \& Sánchez, 2009; Navas \& Sánchez, 2010; Zapata, 2014).

A modo de ejemplo, el trabajo de Aranda \& Castro, orientado a conocer las representaciones raciales en la formación docente inicial, plantea que las actitudes, las concepciones y las representaciones sociales no sólo funcionan como algo constitutivo del discurso de los docentes, sino que incluso cumplen una función importante en modelar las prácticas docentes: "los profesores, inevitablemente modelarán a través de sus prácticas pedagógicas, sus ideas y sus propias concepciones de mundo, además de filtrar el contenido; todo lo cual ha de instalarse en los estudiantes como una forma de representarse el rol docente" (Aranda \& Castro, 2007: 16). En la misma línea, Navas \& Sánchez (2010), quienes analizaron las actitudes hacia la educación multicultural de un grupo de estudiantes, argumentan que las bajas expectativas, los prejuicios y los estereotipos también son parte

Los tres conglomerados son presentados en perspectiva histórica, vale decir, en función de los años de publicación. Así, a) entre los años 2006 y 2012, el tema central fueron las disposiciones docentes; b) entre los años 2010 y 2013, visualizamos temáticas referidas a la discriminación en la escuela, y c) entre los años 2012-2016 los principales tópicos guardan relación con la gestión de la diversidad para una educación intercultural. Por supuesto que el orden cronológico no es estático, y la construcción de rangos permite mostrar las tendencias generales y por tanto los dinamismos del campo de estudio. 
de la labor pedagógica de las docentes, por lo tanto afectarán la relación con los estudiantes inmigrantes $\mathrm{y}$, por consecuencia, el rendimiento de los mismos.

Las disposiciones docentes se presentan como un tema clave en la llegada de los estudiantes inmigrantes a la escuela, pues amarran discursos y prácticas determinadas por parte del profesorado. En ese sentido, algo que emerge del análisis de este ámbito es que la respuesta de la escuela está vinculada a la suma de las subjetividades individuales de los profesionales que la componen. Por lo tanto, la calidad de las respuestas educativas va a obedecer al sentido común espontáneo de la escuela (Rojas, 2014), lo que supone entonces pensar la escuela como un conjunto de unidades aisladas en lugar de pensarla como una auténtica comunidad de práctica (Barton \& Tusting, 2005; Wenger, 1998).

En relación a la discriminación escolar, la entendemos en su sentido amplio como acciones de distinción, exclusión y/o restricción realizadas y que vulneren derechos fundamentales de determinados actores educativos (Mineduc, 2013). Dentro de este conglomerado las investigaciones explicitan las experiencias de racismo vividas principalmente por estudiantes inmigrantes, así como comprender cómo estos estudiantes viven y significan su experiencia de escolarización. Este conjunto de artículos dialoga mayoritariamente con un enfoque de derechos, fijando su atención en las escuelas como contextos socio-comunitarios y denunciando las prácticas racistas que devienen al interior de las instituciones escolares (Hein, 2012; Pavez-Soto, 2010; 2012; Riedemann \& Stefoni, 2015; Stefoni et al., 2016; Tijoux, 2013). Una cita representativa de este corpus documental es la siguiente:

Y estos sujetos-objetos del racismo, en este caso, son los hijos de inmigrantes peruanos por parte de una comunidad educativa que supuestamente entre sus múltiples funciones, debe educar contra el racismo. Es difícil imaginar una educación cívica que no lo considere en sus programas o que solo lo haga para cumplir y tratar los contenidos. [...] Los entrevistados nos han hablado largamente de los barrios de las escuelas, de sus historias de segregación, de las dificultades del hacinamiento y de construcciones inseguras, pero principalmente de la lejanía del Estado (Tijoux, 2013: 303).

Los aspectos nucleares de este segundo conglomerado guardan relación con las barreras que persisten en la escuela para la participación plena de los estudiantes inmigrantes, las cuales, como se menciona en la cita anterior, son parte de una historia de segregación y de construcciones inseguras. Según Pavez-Soto (2010), el ejercicio de derechos de los niños y niñas inmigrantes, posee características específicas que no solo son vinculantes con el contexto legal o político del país, sino que también guarda relación con las dinámicas propias de los establecimientos escolares. En ese sentido, los artículos que componen este conglomerado, interpelan a la escuela en su función transformadora, invitando a los diversos actores de la comunidad escolar a crear las condiciones óptimas de convivencia y de relaciones humanas positivas.

Finalmente la gestión de la diversidad escolar, enfoque importado del ámbito organizacional orientado a la organización de la fuerza de trabajo laboral (Michael, 1977), se ha ido lentamente incorporando a los estudios sobre diversidad cultural en el ámbito escolar, como una forma de resaltar el peso de la dimensión institucional -por sobre otros factores- en la escolarización exitosa de los estudiantes de grupos culturales minoritarios. De esta manera, dentro de este conglomerado los artículos hacen alusión a 
la centralidad que la implementación de medidas organizativas de carácter institucional tiene para la incorporación de los estudiantes inmigrantes. La clásica explicación individualista-culturalista de los problemas escolares es reinterpretada desde la manera en cómo la escuela responde y atiende a la diversidad cultural resultante (Dietz \& Mateos, 2011). Este tercer conglomerado plantea en consecuencia que en la medida que exista una respuesta organizada y comprometida por todos los actores de la escuela, la incorporación de estudiantes inmigrantes se verá favorecida significativamente (Jiménez, 2012, 2014; Jiménez \& Fardella, 2015; Bustos, 2015; Carrasco, Molina \& Baltar, 2013; Donoso, Mardones \& Contreras; 2009; Sánchez, Navas \& Holgado, 2013; Barrios-Valenzuela \& Palou-Julian, 2014;). La siguiente viñeta expresa el espíritu de este tópico:

[...] lejos de variables de naturaleza individual e incluso de origen cultural, el éxito y fracaso escolar del alumnado inmigrante está condicionado fuertemente por los modelos de gestión de la diversidad cultural que implementa la escuela en contextos de multiculturalidad. Esto quiere decir que dependiendo de la forma en que la escuela afronte la llegada y escolarización de alumnado de origen inmigrante, y dependiendo, por ende, de los principios y valores educativos que impregnen sus prácticas pedagógicas, la experiencia educativa del alumnado inmigrante podrá moverse entre el continuo del éxito y del fracaso escolar. Resulta fundamental, por tanto, poder conocer qué modelos existentes en la actualidad pueden ser utilizados por la escuela para gestionar la diversidad cultural del alumnado producto de la incorporación de estudiantes de origen inmigrante (Jiménez, 2014: 411).

Además de estos tres conglomerados centrales, existen artículos que o bien abordan dimensiones no cubiertas por éstos, o en algunos casos incluyen más de uno. Como son igualmente importantes para nuestra revisión, los comentaremos brevemente.

La investigación de Hernández (2016) aborda principalmente las tensiones que ejercen presión al ámbito curricular-pedagógico en aulas con estudiantes inmigrantes, así como las escuelas implementan los cambios y adaptaciones requeridas. Sin embargo y aun cuando el foco está más orientada a la gestión de la diversidad -curricular-, incorpora un análisis sobre las disposiciones que presenta el mismo profesorado que debe poner en prácticas los cambios diseñados. El trabajo de Rubio (2009) en torno al desarrollo de una competencia comunicativa intercultural, plantea una discusión que invita a reflexionar en torno al lugar que la formación inicial de profesores juega en el desarrollo de una ciudadanía e identidades multiculturalmente complejas. Un trabajo en torno a las prácticas pedagógicas del profesorado de historia (Cifuentes, Ebner, Irrazábal, Martínez \& Valdés, 2014), muestra cómo significan estos profesores conceptos tales como interculturalidad, diversidad o exclusión/inclusión y los desafíos que para las prácticas de aula tienen dichos discursos. Pavez-soto (2013) explora las construcciones del concepto de infancia que realizan estudiantes de origen inmigrante, y cómo éstas son tensionadas por las estrategias de aculturación implementadas por las escuelas. Las variables intervinientes en el proceso de elección escolar de familias inmigrantes son abordadas por Joiko \& Vásquez (2016), quienes ofrecen algunas recomendaciones de cara a ajustar este proceso a los planteamientos de una educación intercultural e inclusiva. Finalmente los trabajos de trabajos de Alvites \& Jiménez (2011) y Marín (2014) presentan experiencias de proyectos de intervención pedagógica en los cuales a través del arte terapia y la implementación de un curso de historia, geografía y ciencias sociales sudamericana 
respectivamente. Además de plasmar las dificultades que atraviesan profesores y estudiantes en esta nueva configuración escolar-como discriminación y/o construcción identitaria-, los trabajos reportan el papel que desempeñan los propios contextos escolares como espacios de formación docente en materia de interculturalidad.

\subsection{EL DESDE DÓNDE EN LA INVESTIGACIÓN SOBRE MIGRACIÓN Y ESCUELA: LOS ENFOQUES DE BASE}

Consideramos conveniente interrogar finalmente a los artículos con relación a los enfoques de educación intercultural subyacentes, por dos razones: en primer lugar porque la educación intercultural no es un concepto unívoco sino por el contrario, profundamente polifónico (Barabas, 2014; Dietz \& Mateos, 2011; Muñoz-Sedano, 2010; Walsh, 2007); $\mathrm{y}$, en segundo lugar, por el rol que juega el ámbito académico no sólo en informar, sino también en estructurar el debate contemporáneo y direccionar las políticas públicas en torno a interculturalidad y educación (Pérez Ruiz, 2009). Con el objeto de caracterizar los principales enfoques, vale decir, interrogar los artículos respecto a dónde debería estar puesta la atención o hacia donde deberían orientarse los esfuerzos en materia de interculturalidad (Grant \& Sleeter, 1985), hemos trabajado con el modelo de Dietz \& Mateos (2011) ya que, a nuestro juicio, es una taxonomía que logra conciliar armónicamente planteamientos de origen anglosajón, europeo y latinoamericano en torno a educación intercultural.

Siguiendo la nomenclatura utilizada por Stefoni \& Stang (2017), un primer ámbito de mayor condensación discursiva ${ }^{4}$ en torno a educación intercultural es la de educar para tolerar y/o prevenir el racismo. En la medida que de lo que se trata es de fomentar entre los diferentes actores educativos -aunque principalmente en los estudiantes- el desarrollo de relaciones humanas armónicas basadas en actitudes de respeto a la diferencia cultural, la educación intercultural se sustenta principalmente en el ejercicio de los derechos individuales y gravita por tanto en el ámbito de la convivencia escolar. Educar para tolerar o prevenir el racismo evidenciaría de acuerdo a McLaren (1998) una versión liberal y multiculturalista de entender la educación en contextos de diversidad cultural. Analicemos la siguiente idea extraída de Aranda \& Castro: "Es un hecho que la escuela forma desde y en la diversidad y esta situación debe apoyar el desarrollo de la tolerancia pues exige el respeto a la diferencia, del mismo modo como la vivencia de la justicia requiere la superación de la discriminación. Para estas tareas son significativas las experiencias escolares en una línea democratizante" (Aranda \& Castro, 2007: 9).

La cita es muy ilustrativa de este enfoque en tanto posiciona en el núcleo de la educación intercultural el trabajo con estudiantes pertenecientes al grupo dominante en torno a la tolerancia y la discriminación hacia la diversidad cultural, siendo por tanto el desarrollo de competencias afectivas y actitudinales la manera efectiva de avanzar en la interculturalización de un entorno educativo.

\footnotetext{
$4 \quad$ Metodológica y analíticamente hablando, el procedimiento para determinar el nivel de condensación discursiva consistió en seleccionar todas aquellas citas de los 32 artículos cuya información remitiera a alguno de los enfoques de educación planteados en el modelo de Dietz \& Mateos (2011). Así, de todas las citas seleccionadas (41), 13 de ellas correspondieron a educar para tolerar y/o prevenir el racismo por lo que se constituyó como la primera condensación discursiva, 10 de ellas a educar para transformar correspondiendo a la segunda condensación más significativa, y finalmente, educar para interactuar con 9 citas. El resto de las citas se distribuyeron en el resto de enfoques sin que existiera un patrón recurrente como en los tres casos anteriores.
} 
El trabajo de Navas, Holgado y Sánchez (2009) así como el de Donoso et al. (2009), reafirman la idea que la discriminación es fenómeno/problema que atañe al ámbito de la convivencia escolar, y que la diversidad cultural debe ser trabajada en la medida que supone un factor de enriquecimiento mutuo. Algo distinto son los trabajos de Tijoux (2013) y Stefoni, Stang y Riedemann (2016) los que aportan una mirada más sociológicainstitucional para entender las prácticas discriminatorias, yendo más allá por tanto de explicaciones individuales. Para las autoras, trabajar y prevenir el racismo supone reconocer la dimensión político-ideológica de cualquier proyecto educativo, y desde ese reconocimiento levantar propuestas pedagógicas-curriculares antirracistas que incorporen las relaciones interpersonales de los actores educativos.

Una segunda condensación discursiva -menos significativa que la anterior-es definir el propósito de la educación intercultural como el de educar para transformar. Una educación intercultural transformadora es aquella que se opone a todo tipo de reproducción: lo habitual, lo establecido, lo que suscita y aprecia lo instituido. Una educación intercultural transformadora es altamente deconstructiva en tanto desmantela y/o desarticula lo existente. Es en función de este propósito que el enfoque es autodenominado «multiculturalista crítico» (Kincheloe \& Steinberg, 1999), que en el ámbito educativo se materializa en la necesidad de democratizar las instituciones educativas, proceso que supone deconstruir parte importante de la arquitectura escolar, con el objeto de instituir nuevos modelos, procedimientos y/o categorías explicativas, relacionadas en este caso con la diferencia cultural (Dietz \& Mateos, 2011).

Mientras que educar para tolerar se asocia claramente con una perspectiva liberal de educación intercultural (Jenks, Lee \& Kanpol, 2001), educar para transformar se desplaza levemente hacia perspectivas más críticas (Gorski, 2009). Esto ya que supone un mayor grado de conciencia del rol que los mecanismos ideológicos juegan en el establecimiento de las relaciones de poder, e incluso en la construcción de las diferencias culturales secundarias de los grupos minoritarios y/o de origen inmigrante (Ogbu, 1993). Este mayor grado de conciencia no supone necesariamente un reconstruccionismo social (Grant \& Sleeter, 1985) por lo que no garantiza un cuestionamiento sustantivo del sistema educativo o del funcionamiento escolar.

A través de entrevistas con profesores, el trabajo de Jiménez \& Fardella (2015) muestra los silencios existentes en torno a la idea de representatividad cultural del currículum, pieza clave para la transformación del ámbito curricular-pedagógico hacia una propuesta intercultural. Si bien los autores toman conciencia de este desafío y su potencialidad transformativa, no abordan con mayor precisión cómo iniciar un proceso de rediseño curricular en contextos migratorios. Por su parte, el trabajo de Stefoni, Stang \& Riedemann (2016) el cual plantea la necesidad que las transformaciones que tengan lugar en el espacio escolar en materia de interculturalidad estén en sintonía con las problemáticas que ocurren de manera simultánea en otros espacios sociales. Sin embargo, queda pendiente el precisar cómo podría materializarse una política intercultural en el plano educativo y con énfasis en la condición migratoria. Ambos estudios tienen lo que podríamos llamar una «inspiración reconstruccionista», en tanto cumplen con tomar conciencia del rumbo que deberían seguir las transformaciones educativas.

Un tercer y último núcleo condensa los discursos que ven la interculturalidad como una forma de educar para interactuar, donde no sólo se plantea la heterogeneidad cultural como un componente clave del trabajo pedagógico, sino además en que el desarrollo de 
mecanismos intragrupales de resolución de conflictos resulta central para el desarrollo de competencias interculturales (Dietz \& Mateos, 2011). Estamos frente a un enfoque que a diferencia de los anteriormente expuestos, focaliza la interculturalidad en la dimensión pedagógica de la experiencia educativa, y al mismo tiempo, un trabajo orientado a la totalidad de los estudiantes y no sólo con aquellos pertenecientes a grupos minoritarios. Interactuar es por tanto una actividad central para la interculturalidad y es lo que observamos en la siguiente declaración:

El modelo de educación intercultural que pone el énfasis en el concepto de cultura como un proceso dinámico y de "suma", es, sin duda, el ideal educativo al cual aspirar. Nuestro concepto de educación intercultural incluye también la relación entre culturas dentro del mismo territorio. Así, la educación intracultural sería, obviamente, una característica más dentro del concepto más amplio de interculturalidad [...] La modificación del enfoque pedagógico tiene como objetivo evitar situaciones de conflicto en la escuela, donde el niño-joven de hoy, que será el adulto de mañana, no debería ser abocado a la disyuntiva de optar por una situación de asimilación o exclusión, sino por una integración inclusiva donde todas las personas tengan opción de ser reconocidas y aceptadas y con la libertad de desarrollar su propia identidad cultural (González et al., 2013: 148).

Algo similar observamos en un conjunto de estudios (Navas et al., 2009; Navas \& Sánchez, 2010; Sánchez, Navas \& Holgado, 2013) quienes analizan las actitudes de estudiantes de pedagogía hacia la migración, concluyendo la importancia que los futuros profesores promuevan actividades educativas que promuevan el intercambio cultural. Para ello es fundamental que los programas de formación inicial incorporen espacios para desarrollar sentimientos positivos hacia la diversidad y relaciones íntimas con personas de origen inmigrante. De manera complementaria, en el trabajo de Carrasco, Molina \& Baltar (2013) en escuelas con presencia de estudiantes palestinos, el concepto de mestizaje cultural se ubica en un lugar central, dejando entrever la importancia que la realización de actividades conjuntas, los intercambios, así como las estrategias de contacto, tienen para el desarrollo de una educación intercultural.

Para cerrar este apartado y aun cuando no constituyen una condensación discursiva, hay relaciones entre trabajos y enfoques que conviene incorporar.

El trabajo de Barrios-Valenzuela y Palou-Julián (2014) en torno a la comprensión de las interacciones que estudiantes inmigrantes establecen con sus pares chilenos, es un claro exponente de una educación intercultural que apunta a educar para diferenciar y/o bi-culturalizar (Dietz \& Mateos, 2011). Esto ya que se plantea la escuela como un espacio no sólo para fortalecer dichas interacciones, lo que sería más coherente con el enfoque anterior, sino principalmente para reconocer y estimular el desarrollarlo de la propia identidad cultural de los estudiantes pertenecientes a culturas minoritarias.

Educar para descolonizar a través de la apertura de espacios escolares para producir nuevos saberes, lenguajes y subjetividades (Dietz \& Mateos, 2011), es un enfoque prácticamente ausente de los discursos académicos. Utilizar la presencia de estudiantes inmigrantes como una oportunidad para repensar de manera radical la estructura de las percepciones, conceptualizaciones y prácticas asociadas a la diversidad (MaldonadoTorres, 2007) no ha sido hasta ahora la forma habitual de entender los propósitos de 
la educación intercultural. La excepción la aporta el trabajo de Bustos (2015) el que aborda las experiencias de fricción étnica sufridas por estudiantes de origen inmigrante y pertenecientes a etnias indígenas, en las cuales las situaciones de racismo son vistas como una expresión objetiva de la fuerza de anclaje que el pensamiento colonial tiene sobre los espacios de aprendizaje escolar.

\section{DISCUSIONES}

Como se puede observar del mapeo realizado, la investigación académica en torno a migración y escuela es un campo profundamente heterogéneo teórica, metodológica y epistemológicamente hablando. Más allá de sus diferencias internas, las investigaciones que componen este campo han realizado una indiscutible contribución a la comprensión de esta nueva problemática escolar. Sin duda la investigación académica ha ido «abriendo el camino» por el cual han ido transitando posteriormente otros formatos de investigación educativa. Las investigaciones realizadas permiten que en la actualidad contemos con un diagnóstico lo suficientemente desarrollado -pero no acabado- de diferentes contornos o micro-problemáticas del fenómeno. Esta primera fase, que a nuestro juicio podría denominarse como descriptivo-exploratoria, ha permitido posicionar la nacionalidad como una categoría relevante y preocupante en los procesos de escolarización, y ha actuado a su vez como un dispositivo-analizador (Lourau, 1979), dejando en evidencia la naturaleza monocultural de nuestro sistema educativo. Sin embargo, creemos que es el momento oportuno de que la investigación académica transite hacia otra fase, una fase de mayor paso al acto, una fase aplicada-interventora. Las siguientes discusiones son un aporte a clarificar cómo podríamos pensar dicha etapa.

Comenzaremos con una reflexión. En la investigación que actualmente estamos desarrollando en torno a protocolos escolares de acogida para estudiantes hemos tenido grandes dificultades para acceder a ciertas escuelas, y en las que ya estamos trabajando, para acceder a ciertos profesionales: el caso emblemático ha sido el de dos profesores haitianos que trabajan como traductores en una escuela municipal. En un principio como equipo percibimos una actitud de mucha evasión a la hora de invitarlos a colaborar. Con el tiempo, nuestras explicaciones, seguramente igual que las de muchos otros investigadores, han ido cambiando y hemos podido darnos cuenta que existen reticencias a colaborar con las investigaciones académicas debida a una excesiva saturación de los tiempos y espacios escolares necesarios para llevar a cabo las investigaciones a través de una lógica extractiva, versus una casi nula contribución al mejoramiento en la resolución de los problemas locales que aquejan a las propias escuelas. Esta suspicacia hace que a ojos de las propias escuelas, los investigadores seamos ubicados en los escalafones inferiores con relación al interés e importancia educativa (Contreras et al., 2016).

Consientes que los discursos académicos son tan importantes por lo que dicen como por lo que callan (Essomba, 2005), hemos llamado a estos desafíos los silencios académicos.

Un primer núcleo de silencios lo hemos construido en torno a lo metodológico, ya que interpela justamente el modo de hacer investigación. El primer silencio es incómodo. Siendo la investigación un área clave del sistema educativo nos preguntamos: ¿es la academia un actor realmente transformador del estado actual de la realidad o bien un actor conservador enraizado en prácticas reproductivas? En lo cualitativo, predominan las entrevistas y los 
grupos focales; por el lado cuantitativo, prevalecen las encuestas y las escalas. Ante el desafío de cambios profundos, lo tradicional es sinónimo de estancamiento. En ese sentido, la innovación y la transformación que necesitan -y exigimos a- las escuelas puede verse relegada, al menos en parte, por una investigación conservadora (Echeita, Muñoz, Sandoval \& Simón, 2014). Aquí la invitación no es a mirar hacia fuera como en los tópicos ausentes, sino hacia dentro. El desafío por tanto pasa a nuestro juicio por dejar de investigar en las escuelas y comenzar a realizar investigación con las escuelas, de manera de otorgar una lógica más participativa y democrática a la producción de datos. Como señala Zeichner (2010), una forma de restructurar las relaciones sociales y la economía política en la producción de conocimiento es pedagogizando la investigación. Aun cuando no tenemos un respaldo empírico, nos consta que va en aumento la incorporación de instancias de negociación de resultados en algunas investigaciones en materia de migración y escuela. Nos quedamos con esta pregunta frente a la manera en que estamos generando investigación en torno al fenómeno migratorio desde nuevas propuestas metodológicas y desde formas emancipadoras de relación entre sus participantes. Compartimos con Van Olphen \& Ríos (2004) que las investigaciones educacionales más productivas e incitantes son aquellas que se planean desde la multiplicidad, ya sea de métodos, fuentes de datos o marcos teóricos y que este punto de partida se constituye en un sello de calidad investigativa.

El segundo silencio es corolario del primero y también es inquietante: en un trabajo reciente (Jiménez, Aguilera, Valdés \& Hernández, 2017), hemos podido evidenciar que la gran mayoría de los aportes concretos realizados al trabajo de las escuelas, provienen de organizaciones del ámbito civil, los que se traducen en compensaciones a las deudas del ámbito municipal y estatal. Por tanto, ¿es realmente la investigación que realizamos sobre migración y escuela una contribución real a los procesos de cambio en las escuelas?

Hay dos evidencias necesarias de explicitar. Primero, que los protocolos metodológicos de las investigaciones revisadas tienen un patrón claro: entrar a las escuelas, producir datos, devolver resultados y finalmente difundirlos. Desde este escenario, es evidente que para las escuelas, los investigadores somos personajes periféricos: estamos pero no actuamos con ellos. La primera sensación es que para las escuelas los investigadores son personajes periféricos: observan, pero no actúan. De acuerdo a algunos investigadores en inclusión educativa (Echeita et al., 2014; Parrilla, 2009) una investigación que no contribuya de algún modo al empoderamiento y la emancipación de las personas insertas en procesos de exclusión educativa, no tendría mucho sentido ni valor, llegando incluso a convertirse en un cómplice parcial de dichas exclusiones. Segundo, respecto a las contribuciones declaradas por las investigaciones, también hay una tendencia clara: declarar que el principal propósito de la investigación educativa es contribuir a la transformación social, algo que ya habíamos documentado como algo constitutivo de la identidad académica (Fardella, Sisto \& Jiménez, 2015). Sin embargo, en la mayoría de los casos esto opera transfiriendo al lector la responsabilidad de identificar y posteriormente traducir a formas concretas las contribuciones realizadas. Desde nuestro punto de vista, esto tiene dos supuestos a la base: 1) que las investigaciones son efectivamente consultadas por las personas encargadas de rediseñar las políticas educativas; y 2) que el rediseño de las políticas educativas terminará incidiendo positivamente en la resolución de los problemas detectados en las investigaciones. Consideramos que esta manera de hacer investigación supone una contribución demasiado indirecta e incierta ya que requiere transitar un circuito considerablemente largo para conseguir su propósito. El desafío es por tanto pensar formatos 
de investigación que puedan reportar de manera más directa y oportuna contribuciones al campo de estudio, independiente de si éstas van orientadas al profesorado, las escuelas o a las políticas educativas. Sin duda que un argumento que no podemos desconocer, pero tampoco profundizar en este lugar, radica en el rumbo que ha tomado la investigación académica en los últimos años a propósito del desarrollo de una nueva gubernamentalidad en torno a la producción de conocimiento en Chile y, que incide directamente en los ámbitos escogidos y principalmente en la forma de investigar.

Un segundo núcleo de silencios corresponde a algunos tópicos que pensamos sería importante abordar en futuras investigaciones. El primero de ellos es el rendimiento escolar ya que, salvo datos muy genéricos en torno al desempeño de estudiantes migrantes y chilenos en pruebas estandarizadas, las que señalan que los estudiantes inmigrantes obtienen resultados inferiores que sus pares chilenos (Agencia de Calidad de la Educación, 2014), sería conveniente una mayor comprensión de los factores explicativos de dichas brechas en el aprendizaje. Un segundo ámbito pendiente podría ser el estudio de los efectos y las tensiones que se producen en torno a la distribución, y concretamente la concentración geográfica de estudiantes inmigrantes en determinadas escuelas. En un contexto marcado por la puesta en funcionamiento de la ley de inclusión ${ }^{5}$ que cambia sustancialmente los criterios de selección de las escuelas, convendría preguntarse por la forma en que la población escolar migrante se distribuye en las escuelas. Si bien es un tema en debate, la más reciente investigación en torno al tema en Cataluña-España (Calero \& Escardibúl, 2016) advierte que en escuelas cuyo porcentaje de inmigrantes sobrepasa el $40 \%$, se comienzan a ver afectados negativamente los resultados académicos de inmigrantes y autóctonos. Sería conveniente analizar ello con el fin de contar con evidencia empírica para que desde los municipios puedan tomar decisiones con relación a la distribución escolar de los estudiantes inmigrantes. Un tercer ámbito de estudio pensamos que tiene que ver más bien con un enfoque que con una temática es la necesidad de construir problemas de investigación multidimensionales culturalmente hablando. Esto ya que aun cuando no ha sido objeto de nuestra revisión, nos hemos percatado que son pocos los trabajos que ponen en relación la condición o el estatus migratorio, con otras dimensiones (Bustos, 2015), variables y/o pertenencias de los mismos estudiantes inmigrantes. Esto ya que existen contextos educativos en los que converge lo rural, lo indígena y lo migratorio y más que aislar la condición migrante, convendría ponerla en diálogo con estas otras de manera de repensar el trasfondo monocultural que persiste $-\mathrm{y}$ resiste- a la base de nuestro modelo educativo.

Un cuarto ámbito pendiente de estudio que consideramos importante plantear es en torno a profesores migrantes insertos en nuestro sistema educativo, específicamente a aquellos que son incorporados a las escuelas para facilitar el aprendizaje de estudiantes palestinos, sirios o haitianos, por ejemplo- que no manejan la lengua vehicular de nuestras escuelas. Ha sido nuestra experiencia de campo en una escuela municipal de Estación Central donde hemos visto en acción a profesores haitianos haciendo de intérpretes para los estudiantes recién llegados. Nuestra conclusión es que son prácticas bastante discrecionales y muchas veces basadas en relaciones de improvisación con los profesores disciplinares.

Para una revisión crítica con relación a la escasa presencia de la condición migratoria, se sugiere leer la columna de Sara Joiko "Políticas migratorias de Chile en el área educativa: La Ley de Inclusión", disponible en:http://www.elmostrador.cl/ noticias/opinion/2016/04/24/politicas-migratorias-de-chile-en-el-area-ducativa-la-ley-de-inclusion/ 
Esto abre un conjunto de preguntas acerca del rol de estos profesores, de las formas de trabajo con sus pares, de los marcos regulatorios que existen sobre su trabajo o en torno a las prioridades lingüísticas de una escuela. Todas ellas suponen en la actualidad lagunas que convendría comenzar a saldar de manera empírica. Un quinto ámbito, que en nuestro caso como equipo estamos abordando en este momento, tiene que ver con la aparición de nuevos actores escolares, específicamente la puesta en funcionamiento de protocolos y programas de acogida que más allá de simples documentos, terminan actuando como verdaderos dispositivos (Harré, 2002; Sisto \& Zelaya, 2013) que guían la acción educativa. Este tipo de dispositivos, aun no impuestos y requeridos por la política educativa vigente, están contribuyendo a transformar positivamente la experiencia escolar en materia de incorporación de estudiantes inmigrantes (Palaudàrias \& Garreta, 2008). Si bien ya hay un trabajo incipiente al respecto, convendría profundizar en la compresión en el uso y efectos que estos sujetos/objetos producen en el quehacer de las escuelas.

Para cerrar este ámbito de silencios no podemos dejar de mencionar, en el marco de las transformaciones que el fenómeno migratorio ha tenido en nuestro país, la importancia de comenzar a tiempo estudios sobre la experiencia educativa de estudiantes inmigrantes de segunda generación ${ }^{6}$, o dicho de manera más justa, estudiantes de padres inmigrantes. Incluimos también en este ámbito la denominada generación 1.5 que contempla estudiantes que, si bien no han nacido en el país de acogida, lo han hecho a muy temprana edad. Aquí convendría explorar no sólo la diversidad de procesos identitarios e identificaciones culturales en juego (Essomba, 2006), sino además las implicancias educativas de estos procesos no exentos de tensiones y conflictos. En un contexto investigativo marcado por el reconocimiento de los déficits escolares y por privilegiar discursivamente lo que las escuelas/profesores no hacen o no tienen, investigaciones que tengan como punto de partida los acervos educativos que tanto profesores y equipos directivos han ido desarrollando y acumulando. Así como el programa Funds of Knowledge (Esteban-Guitart \& Vila, 2013; Jovés, Siqués \& Esteban-Guitart, 2015) ha tomado este camino con los estudiantes inmigrantes rescatando sus saberes y conocimientos no escolares para darles un uso pedagógico, uno de los desafíos internacionales es justamente trasladar esta mirada hacia los profesores (Llopart \& Esteban-Guitart, 2017). Creemos que los profesores sí tienen un cúmulo de conocimiento implícito sobre la escolarización de estudiantes inmigrantes, sobre todo en aquellas escuelas que en la actualidad han ido adquiriendo una suerte de tradición en el tema, y que sin lugar a dudas convendría documentar a través de investigación educativa. El trabajo realizado por la Fundación Superación de la Pobreza (2016) con seis escuelas de la región metropolitana es un excelente exponente de una investigación educativa desde y para las propias escuelas, y un indiscutible ejemplo del rol compensador del ámbito civil del que hablamos anteriormente.

Finalmente y con relación al tercer núcleo de silencios, resulta oportuno hacer una primera conclusión global que surge del trabajo realizado y es que globalmente hablando, las investigaciones en torno a migración y escuela en Chile se han realizado sin poner en un lugar central el peso que un enfoque neoliberal -centrado en la competitividad, cumplimiento de estándares y fiscalización- tiene sobre el modo de educar en las escuelas. Muy centrada en la idea de reforma y mejora (Gentili, 2011) esta perspectiva supone no

\footnotetext{
6 La categoría fue acuñada por la socióloga española Rosa Aparicio y ha sido objeto de críticas y controversias, en la medida que resalta la condición migrante de estos estudiantes por sobre su nacionalidad (el país de nacimiento).
} 
cuestionar algunos de las propiedades de nuestro sistema educativo actual (competencia, estandarización de procesos, rendición de cuentas, mejoramiento continuo) y que pueden estar a la base de las desigualdades que afectan a los estudiantes de origen inmigrante o bien a las escuelas en las que se escolarizan. En este sentido, sin poner en duda la utilidad práctica que las recomendaciones puedan tener para el mejoramiento de las diferentes dimensiones del quehacer escolar, pareciera que hasta ahora las investigaciones han tenido un bajo protagonismo en cuestionar las reglas del juego de nuestro sistema educativo. Como investigadores creemos que esto es central a la hora de contribuir a la transformación educativa, generando a fin de cuentas una complicidad ideológica crítica con dicho sistema (Bahía Bock, 2003). Esto interpela por tanto uno de los principales y más recurrentes propósitos de la investigación académica reportada en la propia literatura revisada, que es justamente el de aportar a la transformación social.

\section{REFERENCIAS BIBLIOGRÁFICAS}

Abett de la Torre, P. (2011). La migración vivenciada en el espacio escolar: la experiencia de alumnado extranjero en una escuela pública de Santiago. Paulo Freire, revista de pedagogía crítica, 10 (9), 113-126. Recuperado el 30 de mayo de 2017 desde http://bibliotecadigital. academia.cl/handle/123456789/3118

Agencia de Calidad de la Educación (2014). Informe Nacional Resultados Chile PISA 2012. Santiago: AGCE.

Alvites, L. \& Jiménez, R. (2011). Niños y niñas migrantes, desafío pendiente. Innovación educativa en escuela de Santiago de Chile. Synergies Chili, 7, 121-136. Recuperado el 30 de mayo de 2017 desde https://gerflint.fr/Base/Chili7/alvites.pdf

Aranda, V. \& Castro, F. (2007). Representaciones del imaginario racial en pedagogía. Revista electrónica diálogos educativos, 7(13), 1-19. Recuperado el 30 de mayo de 2015 desde http:// www.umce.cl/ dialogos/n13_2007/aranda.swf

Aranda, V. (2011). Reflexión y análisis de políticas y prácticas innovadoras a la luz de las representaciones sociales y de la necesidad de una educación intercultural en la formación inicial docente. Estudios pedagógicos, 37(2), 301-314. DOI: https://dx.DOI.org/10.4067/S071807052011000200018

Bahía Bock, A. (2003). Psicología de la Educación: Complicidad Ideológica. En A. Bahía Bock, A. Checcia \& M. Rebello De Souza (Eds.). Psicología Escolar: Teorías críticas. (pp. 79-103). Sao Paulo: Casa do Psicólogo.

Barabas, A. (2014). Multiculturalismo, pluralismo cultural e interculturalidad en el contexto de América Latina: la presencia de los pueblos originarios. Configurações, Revista de Sociología, 14, 11-24. Recuperado el 30 de mayo de 2017 desde https://journals.openedition.org/ configuracoes/2219\#quotation

Barrios-Valenzuela, Ll. \& Palou-Julián, Berta. (2014). Educación intercultural en Chile: la integración del alumnado extranjero en el sistema escolar. Educación y Educadores, 17(3), 405-426. DOI: https://dx.DOI.org/10.5294/edu.2014.17.3.1

Barton, D. \& Tusting, K. (2005). Beyond communities of practice. Language, power and social context. New York: Cambridge University Press.

Bravo, R. (2012). Inmigrantes en la escuela chilena: ciertas representaciones para ciertas políticas en educación. Revista Latinoamericana de Inclusión Educativa, 6(1), 39-52. Recuperado el 30 de mayo de 2017 desde http://www.rinace.net/rlei/numeros/vol6-num1/art2.pdf.

Bustos, C. (2015). Historia y memoria: una exigencia a la política pública para la educación 
Estudios Pedagógicos XLIV, $\mathrm{N}^{\circ}$ 3: 173-191, 2018

GEOGRAFÍAS DE LA INVESTIGACIÓN ACADÉMICA SOBRE MIGRACIÓN Y ESCUELA: VOCES, SILENCIOS Y

PROSPECTIVAS DE NUESTRA PROFESIÓN

intercultural en las escuelas de chile. El Ágora U.S.B., 15(2), 401-417. Recuperado el 30 de mayo de 2017 desde http://www.scielo.org.co/scielo.php?script=sci_arttext\&pid=S1657$80312015000200005 \& \operatorname{lng}=\mathrm{es} \& \operatorname{tln} \mathrm{=}=\mathrm{es}$.

Calero, J. \& Escardibúl, J. (2016). La adquisición de competencias en estudiantes autóctonos e inmigrantes. Barcelona: Observatorio Social La Caixa.

Cárdenas, M. (2006). "Y verás cómo quieren en Chile...”: Un estudio sobre el prejuicio hacia los inmigrantes bolivianos por parte de los jóvenes chilenos. Última década, 14(24), 99-124. DOI: https://dx.DOI.org/10.4067/S0718-22362006000100006

Carrasco, C., Molina, M. \& Baltar, M.J. (2013). Inmigración, infancia e integración socioeducativa: un estudio etnográfico sobre niños palestinos en Chile. Revista Chilena de Antropología, 27, 141-16. Recuperado el 30 de mayo de 2017 desde http://www.revistadeantropologia.uchile.cl/ index.php/RCA/article/viewFile/27361/29002

Carrillo, C. (2014). Reflexión en torno a las construcciones simbólicas de los/las profesores/ras respecto de la inclusión educativa de hijos e hijas de inmigrantes en el sistema educativo chileno. TS Cuadernos de Trabajo Social, 11, 43-56. Recuperado el 30 de mayo de 2017 desde http:// www.tscuadernosdetrabajosocial.cl/index.php/TS/article/view/12/12

Cifuentes, M., Valdés, C., Martínez, T., Irrazábal, S. \& Enber, J. (2014). El profesorado de historia y ciencias sociales frente al desafío de educar en contextos multiculturales. Paulo Freire. Revista de pedagogía crítica, 16, 133-147. Recuperado el 30 de mayo de 2017 desde http:// bibliotecadigital.academia.cl/handle/123456789/3808

Cisternas, T. (2011). La investigación sobre formación docente en chile. Territorios explorados e inexplorados. Calidad en la educación, 35, 131-164. DOI: http://dx.DOI.org/10.4067/S071845652011000200005

Contreras, P., Assaél, J., Acuña, F., Santa Cruz, E., Campilay, B. \& Pujadas, B. (2016). Construyendo saber etnográfico: reflexiones a partir de la experiencia de campo en instituciones escolares. Athenea Digital, 16(3), 55-79. Recuperado el 30 de abril de 2017 desde http://www.redalyc.org/ articulo.oa? id $=53748488003$

Dietz, G. \& Mateos, S. (2011). Interculturalidad y educación intercultural en México. Un análisis de los discursos nacionales e internacionales en su impacto en los modelos educativos mexicanos. México: Secretaría de Educación Pública.

Donoso, A., Mardones, P. \& Contreras, R. (2009) Propuestas y desafíos a partir de la experiencia de una escuela con migrantes en el Barrio Yungay, Santiago de Chile. Revista Docencia, 37, 56-62. Recuperado el 28 de julio de 2016 desde http://www.revistadocencia.cl/pdf/20100730183415. pdf

Echeita, G., Muñoz, Y., Sandoval, M. \& Simón, C. (2014). Reflexionando en voz alta sobre el sentido y algunos saberes proporcionados por la investigación en el ámbito de la educación inclusiva. Revista Latinoamericana de Inclusión Educativa, 8(2), 25-48. Recuperado el 20 de marzo de 2017 desde http://www.rinace.net/rlei/numeros/vol8-num2/art1.pdf

Essomba, M. (2006). Liderar escuelas interculturales e inclusivas. Equipos directivos y profesorado ante la diversidad cultural y la inmigración. Barcelona: Graó.

(2005). L'atenció a la diversitat a Catalunya. Vic: Eumo Editorial

Esteban-Guitart, M. \& Vila, I. (2013). Experiencias en educación inclusiva. Vinculación familia, escuela y comunidad. España: Horsori.

Fardella, C., Sisto, V. \& Jiménez, F. (2015). Nosotros los académicos. Narrativas identitarias y autodefinición en la universidad actual. Universitas Psychológica, 14(5), 1625-1636. DOI: http://dx.DOI.org/10.11144/Javeriana.upsy14-5.nani

Fundación Superación de la Pobreza (2016). Educación e Interculturalidad en Escuelas Públicas. Orientaciones desde la Práctica. Santiago: FSP.

Gentili, P. (2011). Pedagogía de la igualdad. Ensayos contra la educación excluyente. Buenos Aires: Siglo XXI. 
Glaser, B. \& Strauss, A. (1967) Discovery of grounded theory. Chicago: Aldine.

González, O., Berríos, Ll. \& Buxarrais, M. R. (2013). La sensibilidad del profesorado hacia el modelo de educación intercultural: Necesidades, situación actual y propuesta de un instrumento de medida. Estudios pedagógicos, 39(2), 147-164. DOI: https://dx.DOI.org/10.4067/S071807052013000200010

Gorski, P. (2009). What we're teaching teachers: An analysis of multicultural teacher education coursework syllabi. Teaching and Teacher Education, 25, 309-318. DOI: https://doi. org/10.1016/j.tate.2008.07.008

Grant, C. \& Sleeter, C. (1985). The Literature on Multicultural Education: review and analysis. Educational Review, 37(2), 97-118. Recuperado el 20 de abril 2016 desde https://www. tandfonline.com/doi/abs/10.1080/0305569860120104

Harré,R.(2002). Material Objectsin SocialWorlds. Theory, Culture \& Society, 19(4), 23-33. Recuperado el 12 de abril de 2017 desde http://journals.sagepub.com/doi/10.1177/026327640201900502

Hein, K. (2012). Migración y transición: hijos de inmigrantes de origen latinoamericano en su transición de la escuela al trabajo en Chile. Si Somos Americanos, 12(1), 101-126. DOI: https:// dx.DOI.org/10.4067/S0719-09482012000100005

Hernández, A. (2016). El currículo en contextos de estudiantes migrantes: Las complejidades del desarrollo curricular desde la perspectiva de los docentes de aula. Estudios pedagógicos, 42(2), 151-169. DOI: https://dx.DOI.org/10.4067/S0718-07052016000200009

Jenks, C., Lee, J. y Kanpol, B. (2001). Approaches to Multicultural Education in Preservice Teacher Education: Philosophical Frameworks and Models for Teaching. The Urban Review, 33(2), 87-105. Recuperado el 30 de noviembre de 2016 desde https://link.springer.com/ article/10.1023/A:1010389023211

Jiménez, F. (2014). Modelos de gestión de la diversidad cultural para la escolarización de alumnado inmigrante en las escuelas chilenas: Desafíos para la interculturalidad actual. Estudios pedagógicos, 40(2), 409-426. DOI: https://dx.DOI.org/10.4067/S0718-07052014000300024

. (2012). Violencia Escolar en Contextos Educativos Multiculturales: Una Aproximación Desde los Modelos de Gestión de la Diversidad Cultural. Psicoperspectivas, 11(2), 8-30. DOI: https://dx.DOI.org/10.5027/psicoperspectivas-Vol11-Issue2-fulltext-205

Jiménez, F., Aguilera, M., Valdés, R. \& Hernández (2017). Migración y escuela: Análisis documental en torno a la incorporación de inmigrantes al sistema educativo chileno. Psicoperspectivas, 16(1), 105-116. DOI: 10.5027/psicoperspectivas-vol16-issue1-fulltext-940

Jiménez, F. \& Fardella, C. (2015). Diversidad y rol de la escuela. Discursos del profesorado en contextos educativos multiculturales en clave migratoria. Revista Mexicana de Investigación Educativa, 20(65), 419-441. Recuperado el 30 de mayo desde 2017 desde http://www.redalyc. org/articulo.oa?id=14035408005

Joiko, S. \& Vásquez, A. (2016). Acceso y elección escolar de familias migrantes en Chile: No tuve problemas porque la escuela es abierta, porque acepta muchas nacionalidades. Calidad en la educación, 45, 132-173. DOI: https://dx.DOI.org/10.4067/S0718-45652016000200005

Jovés, P., Siqués, C. \& Esteban-Guitart, M. (2015). The Incorporation of Funds of Knowledge and Funds of Identity of Students and their Families into Educational Practice. A Case Study from Catalonia, Spain. Teaching and Teacher Education, 49, 68-77. DOI: 10.1016/j.tate.2015.03.001

Kincheloe, J. \& Steinberg, S. (1999). Repensar el multiculturalismo. Barcelona: Octaedro.

Llopart, M. \& Esteban-Guitart, M. (2017). Strategies and resources for contextualising the curriculum based on the funds of knowledge approach: a literature review. The Australian Educational Researcher, 44, 1-20. DOI: 10.1007/s13384-017-0237-8

Lourau, R. (1979). El análisis institucional. Argentina: Amorrortu Editores.

Maldonado-Torres, N. (2007). On the Coloniality of Being: contributions to the development of a concept. Cultural Studies, 21(2), 240-270. Recuperado el 30 de abril de 2016 desde https://www. tandfonline.com/doi/abs/10.1080/09502380601162548 
Estudios Pedagógicos XLIV, $\mathrm{N}^{\circ}$ 3: 173-191, 2018

GEOGRAFÍAS DE LA INVESTIGACIÓN ACADÉMICA SOBRE MIGRACIÓN Y ESCUELA: VOCES, SILENCIOS Y PROSPECTIVAS DE NUESTRA PROFESIÓN

Marín, J. (2014). Ayni: Por una infancia sin fronteras. Arteterapia con hijos de migrantes en el norte de Chile. Arteterapia. Papeles de arteterapia y educación artística para la inclusión social, 9, 61-72. DOI: http://dx.DOI.org/10.5209/rev_ARTE.2014.v9.47482

McLaren, P. (1998). Multiculturalismo revolucionario. Pedagogías de disensión para el nuevo milenio. Madrid: Siglo XXI

Michael, S. (1977). Models of Multiculturalism: implications for the twenty-first century leaders. European Journal of Intercultural Studies, 8(3), 37-41. Recuperado el 15 de mayo de 2017 desde https://www.tandfonline.com/doi/abs/10.1080/0952391970080302

Ministerio de Educación (2013). Discriminación en el contexto escolar. Orientaciones para promover una escuela inclusiva. Santiago: Mineduc.

Moreno, K. \& Oyarzún, C. (2013). ¿Interculturalidad en el sistema educacional chileno?: Representaciones sociales de la integración de estudiantes extranjeros en dos establecimientos educacionales municipales. Revista Electrónica de Psicología Iztacala, 16(3), 764-790. Recuperado el 20 de marzo de 2017 desde http://www.revistas.unam.mx/index.php/repi/article/ view/41789/37949

Muñoz-Sedano, A. (2010). Hacia una educación intercultural: enfoques y modelos. Contrastes, Revista Cultural, 58, 49-57. Recuperado el 17 de agosto de 2016 desde https://revistas.ucm.es/ index.php/RCED/article/viewFile/RCED9898220101A/17334

Navas, L., Holgado, F. \& Sánchez, A. (2009). Predicción de los estereotipos académicos ante los estudiantes inmigrantes. Horizontes Educacionales, 14, 37-47. Recuperado el 30 de junio de 2016 desde http://www.redalyc.org/articulo.oa?id=97915161004

Navas, L. \& Sánchez, A. (2010). Actitudes de los Estudiantes de Pedagogía de las Regiones del Bío Bío y la Araucanía de Chile hacia la Presencia de Niños Inmigrantes en la Escuela: Análisis Diferenciales. Psykhe, 19(1), 47-60. DOI: https://dx.DOI.org/10.4067/S071822282010000100004

Ogbu, J. (1993). Etnografía escolar. Una aproximación a nivel múltiple. En H. Velasco, F. J. García, y Á. Díaz de Rada (Eds.), Lecturas de antropología para educadores: el ámbito de la antropología de la educación y de la etnografía escolar (pp. 145-175). Madrid: Editorial Trotta.

Palaudàrias, J. \& Garreta, J. (2008). La acogida del alumnado de origen inmigrante: un análisis comparado desde la situación en Cataluña. Revista Española de Educación Comparada, 14, 49-78. Recuperado el 18 de abril de 2017 desde http://www.sc.ehu.es/sfwseec/reec/reec14/ reec1402.pdf

Parrilla, A. (2009). ¿Y si la investigación sobre inclusión no fuera inclusiva? Reflexiones desde una investigación biográfico-narrativa. Revista de Educación, 349, 101-117. Recuperado el 14 de septiembre de 2017 desde http://www.revistaeducacion.mec.es/re349/re349_05.pdf

Pavez-Soto, I. (2013). Los significados de ser niña y niño migrante: conceptualizaciones desde la infancia peruana en Chile. Polis, 12(35), 183-210. DOI: https://dx.DOI.org/10.4067/S071865682013000200009

. (2012). Inmigración y racismo: experiencias de la niñez peruana en Santiago de Chile. Si Somos Americanos, 12(1), 75-99. DOI: https://dx.DOI.org/10.4067/S0719-09482012000100004

(2010). Los derechos de las niñas y niños peruanos en Chile: La infancia como un nuevo actor migratorio. Revista Enfoques, 8(12), 27 - 51. Recuperado el 30 de junio de 2016 desde http://www.revistaenfoques.cl/index.php/revista-uno/article/view/151

Perez-Ruiz, M. (2009). ¿De qué hablamos cuando nos referimos a lo intercultural? Reflexiones sobre su origen, contenidos, aportaciones y limitaciones. En M. Pérez Ruiz, L. Valladares de la Cruz \& M. Zárate (Eds.), Estados plurales: los retos de la diversidad y la diferencia (pp. 251-288). México: UNAM.

Riedemann, A. \& Stefoni, C. (2015). Sobre el racismo, su negación, y las consecuencias para una educación anti-racista en la enseñanza secundaria chilena. Polis, 14(42), 191-216. DOI: https:// dx.DOI.org/10.4067/S0718-65682015000300010 
Rojas, M. T. (2014). Las creencias docentes: Delimitación del concepto y propuesta para la investigación. Revista Electrónica Diálogos Educativos, 14(27), 89-112. Recuperado el 15 de julio de 2016 desde de http://www.dialogoseducativos.cl/revistas/n27/rojas

Rubio, M. (2009). El desarrollo de la competencia comunicativa intercultural en la formación inicial docente. Estudios pedagógicos, 35(1), 273-286. DOI: https://dx.DOI.org/10.4067/S071807052009000100017

Sánchez, A., Navas, L. \& Holgado, P. (2013). Inmigración y educación intercultural en la formación inicial docente. Estudios pedagógicos, 39(1), 239-251. DOI: https://dx.DOI.org/10.4067/S071807052013000100014

Serra, C. (2004). Etnografía escolar, etnografía de la educación. Revista de Educación, 334, 165-176. Recuperado el 30 de abril de 2015 desde http://www.revistaeducacion.mec.es/re334/re334_11. pdf

Sisto, V. \& Zelaya, V. (2013). La etnografía de dispositivos como herramienta de análisis y el estudio del managerialismo como práctica local. Universitas Psychologica, 12(4), 1345-1354. DOI: 10.11144/Javeriana.UPSY12-4.edha

Sleeter, C. (2013). Teaching for social justice in multicultural classroom. Multicultural Education Review, 5(2), 1-18. Recuperado el 14 de noviembre de 2016 desde https://eric. ed.gov/?id=EJ1131396

Stefoni, C. \& Stang, F. (2017). La construcción del campo de estudio de las migraciones en Chile: notas de un ejercicio reflexivo y autocrítico. Íconos. Revista de Ciencias Sociales, 58, 109-129. DOI: http://dx.DOI.org/10.17141/iconos.58.2017.2477

Stefoni, C., Stang, F. \& Riedemann, A. (2016). Educación e interculturalidad en Chile: Un marco para el análisis. Estudios internacionales, 48(185), 153-182. DOI https://dx.DOI.org/10.5354/07193769.2016.44534

Tijoux, M. E. (2013). Las escuelas de la inmigración en la ciudad de Santiago: Elementos para una educación contra el racismo. Polis, 12(35), 287-307. DOI: http://dx.DOI.org/10.4067/s071865682013000200013

Van Olphen, M. \& Ríos, F. (2004). Al servicio de la justicia social: conocimientos, destrezas y actitudes para los nuevos tiempos. Psicoperspectivas, 3(1), 151-171. Recuperado el 30 de marzo de 2017 desde http://www.psicoperspectivas.cl/index.php/psicoperspectivas/article/view/16

Villalobos, C. \& Carrillo, C. (2014). Inclusión/exclusión de estudiantes inmigrantes en Chile. La emergencia de un fenómeno sociocultural en el sistema educativo chileno. I Bienal Latinoamericana de Infancias y Juventudes, 53(9), 1689-1699.

Villegas, A. (2007). Dispositions in teacher education: A look at social justice, Journal of Teacher Education, 58(5), 370-380. Recuperado el 15 de junio de 2016 desde https://eric. ed.gov/?id=EJ778023

Walsh, K. (2007). Interculturalidad, colonialidad y educación, Revista Educación y Pedagogía, 19(48), 25-35. Recuperado el 19 de noviembre de 2016 desde http://www.flacsoandes.edu.ec/ sites/default/files/agora/files/1265909654.interculturalidad_colonialidad_y_educacion_0.pdf

Wenger, E. (1998). Communities of practice. Learning, meaning, and identity. New York: Cambridge University Press.

Wolcott, H. (1993). La intención etnográfica. En A. Díaz de Rada, H. Velasco \& J. García (Eds.), Lecturas de antropología para educadores. El ámbito de la antropología de la educación y de la etnografía escolar (pp. 127-144). Madrid: Trotta.

Zapata, R. (2014). Diversidad cultural en la formación del futuro profesorado en América Latina. Necesidades y perspectivas. Revista Electrónica Interuniversitaria de Formación del Profesorado, 17(2), 219-234. DOI: http://dx.DOI.org/10.6018/reifop.17.2.197611

Zeichner, K. (2010). La formación del profesorado y la lucha por la justicia social. Madrid: Morata. 
